\title{
FACT - Highlights from more than Seven Years of Unbiased Monitoring at TeV Energies
}

\author{
Daniela Dorner*, for the FACT Collaboration ${ }^{\dagger}$ \\ University of Würzburg \\ E-mail: dorner@astro.uni-wuerzburg.de
}

The First G-APD Cherenkov Telescope (FACT) has been monitoring TeV-blazars since October 2011. Thanks to its unbiased observing strategy, the robotic operation and the usage of solid state photosensors (SiPM, aka G-APDs), the instrument's duty cycle has been maximized and the observational gaps minimized, providing an unprecedented data sample of more than 13'800 hours of physics data, of which more than 2600 hours were taken in the past 12 months.

Results of an automatic quick-look analysis are published with low latency on an open-access website. Based on this, 98 alerts and 11 astronomer's telegrams have been issued in more than five years, triggering a variety of multi-wavelength studies. Target-of-opportunity programs with $\mathrm{X}$-ray satellites are in place.

For example, combined gamma-ray observations (FACT, Fermi-LAT, HESS) on Mrk 501 in 2014 have been used to constrain the underlying physics processes analysing the shape of flux distributions on different time scales.

A trigger to MAGIC on a high state of 1ES 2344+51.4 in 2016 reveals a very hard spectrum. The combined observations are used to study the source's extreme behaviour.

Studying the long-term multi-wavelength behaviour of Mrk 421, more than 30 flares have been found in 5.5 years of data. Correlating different wavelengths, delays are determined and models are constrained.

In addition to multi-wavelength studies, the unprecedented, unbiased data sample at TeV energies provides a unique chance to study the duty cycle and variability behaviour of the sources. Based on this, a search for a periodic signal has been performed.

36th International Cosmic Ray Conference -ICRC2019-

July 24th - August 1st, 2019

Madison, WI, U.S.A.

\footnotetext{
* Speaker.

${ }^{\dagger}$ for collaboration list and acknowledgements see PoS(ICRC2019)1177
} 


\section{Blazars}

Active Galactic Nuclei (AGNs) are highly variable sources emitting radiation along the electromagnetic spectrum. In the centre, they harbour a black hole surrounded by an accretion disk and a dusty torus. Perpendicular to the accretion disk, AGNs feature particle outflows, so-called jets. Blazars are a subclass of AGNs, for which the jet is oriented towards the observer.

Broad-band Energy Spectrum Like for other AGNs, the spectral energy distribution (SED) of blazars features two bumps, with the low energy bump in the optical to X-ray regime and the high energy bump in the gamma-ray regime. Blazars that do not show emission or absorption lines are named BL Lac type objects. They are subclassified according to their peak energy. For the observation at $\mathrm{TeV}$ energies, the class of high-frequency peaked BL Lac type objects (HBLs) is the most interesting, as they have their high-energy peak in the $\mathrm{GeV}$ to $\mathrm{TeV}$ energy range.

While the low-energy peak is understood as synchrotron emission of an electron distribution in the jet, the origin of the high-energy peak is still under debate. A variety of models explain the high-energy peak with different mechanisms and suggest different emission locations. The most common models attribute the high-energy emission to either inverse Compton scattering of electrons or pion production by hadrons. In the former case, a correlation between high-energy and synchrotron emission is expected, while in the latter case, neutrino emission is predicted. Therefore, multi-wavelength (MWL) and multi-messenger observations are important to discern the emission mechanism and draw conclusions on the emission region. Due to the featureless shape of the spectra and the high number of model parameters, usually most models can explain the observed spectra. Only additional as constraints as the size of the emission region obtained from variability measurements or the temporal evolution of the SED may solve this ambiguity.

Variability One of the most distinct features of blazars is their extreme variability on timescales from minutes [1] to years [2,3]. Depending on the type of underlying process, different shapes of the flux distributions are expected [4]. Apart from that, flux distributions can be used to classify flux states and determine the source's duty cycle.

Correlating different wavelengths and messengers, conclusions on the underlying physics can be drawn. Due to the variability, it is crucial to have continuous light curves in all wavelengths for these studies. While a large number of optical telescopes is available and can provide long-term light curves in the visible range of the energy spectrum, other wavelengths are more limited due to the few available instruments and the competing physics questions that are addressed by the instruments.

\section{Long-Term Monitoring}

\subsection{Multi-Wavelength Context}

In the optical and radio regime, a variety of instruments and monitoring programs are available $[5,6]$. In the high-energy regime, the situation is slightly different due to the limited number of instruments. In X-rays, on the one hand pointed instruments with high sensitivity (e.g. SwiftXRT, XMM-Newton, NuSTAR) perform a variety of different observations and physics cases. For 
those, only very limited time is available for monitoring. On the other hand, all-sky monitoring is available (e.g. Swift-BAT, MAXI) with limited sensitivity which results in limited time resolution.

At GeV gamma rays, the Large Area Telescope (LAT) on-board the Fermi satellite is scanning the sky. Results from this all-sky monitoring are discussed in detail in [7]. Also monitoring programs combining different energy ranges are available [8].

Observations in gamma rays are important to study the high energy bump of blazars. For HBLs, the rising part is seen at $\mathrm{GeV}$ energies, and the $\mathrm{TeV}$ range covers the declining part.

\subsection{Monitoring in Very High Energy Gamma Rays}

At very high energies (VHE; $>100 \mathrm{GeV}$ ), the situation with respect to monitoring is similar as in X-rays with only a limited number of instruments available. Different observational techniques feature different advantages. While imaging air Cherenkov telescopes (IACTs) do pointed observations with high sensitivity, water-Cherenkov instruments monitor a large fraction of the sky with lower sensitivity independent of weather and sun. The complementarity of the two techniques is discussed at the example of FACT and HAWC in $[9,10]$. Although large IACTs like MAGIC, H.E.S.S. and VERITAS have monitoring programs, they study a variety of physics cases, limiting the time, these instruments can dedicate to the monitoring of known sources. Observing a set of sources for a total of only 100-250 hours per year, results in an observation time of 20-100 hours per source per year, which results in either sparse sampling or very short individual exposures.

Further limitations are introduced by the usage of photo-multiplier-tubes (PMTs), as their gain degrades when exposed to too much light, limiting the observation time. 1000 hours of dark night observation time are available per year. Observing with a bit of moon light, the observation time can be extended by 300 hours to 800 hours introducing special observational setups.

In addition to the monitoring, often the observations are extended in case of high flux or additional observations are scheduled, in case an alert on a source is received. This introduces a bias in the result towards higher fluxes.

\subsection{FACT Monitoring Program}

In TeV gamma rays, the First G-APD Cherenkov Telescope (FACT) [11] is monitoring bright blazars since October 2011. FACT is located at $2200 \mathrm{~m}$ a.s.l. in the Observatorio del Roque de los Muchachos on the Canary Island La Palma.

Minimized Gaps and Maximized Duty Cycle The excellent and stable performance of FACT's SiPM camera allows for consistent measurements and reduces the gaps around full moon $[12,13]$. Minimizing the gaps and maximizing the duty cycle of the instrument, in the last 12 months, 2602 hours $^{1}$ of physics data have been collected. In total, 13' 830 hours $^{1}$ of physics data have been recorded. Find a summary of the amount of physics data taken so far in Table 1.

Maximized Data Taking Efficiency Starting from summer 2012, remote operation was carried out routinely. The data taking procedure was automatized during 2012 and 2013 in hard- and software. This is reflected in an increasing data taking efficiency (see Fig. 1). Thanks to the excellent performance of the SiPMs and the feedback system used in FACT to keep the gain stable [12],

\footnotetext{
${ }^{1}$ status 29.6 .2019
} 

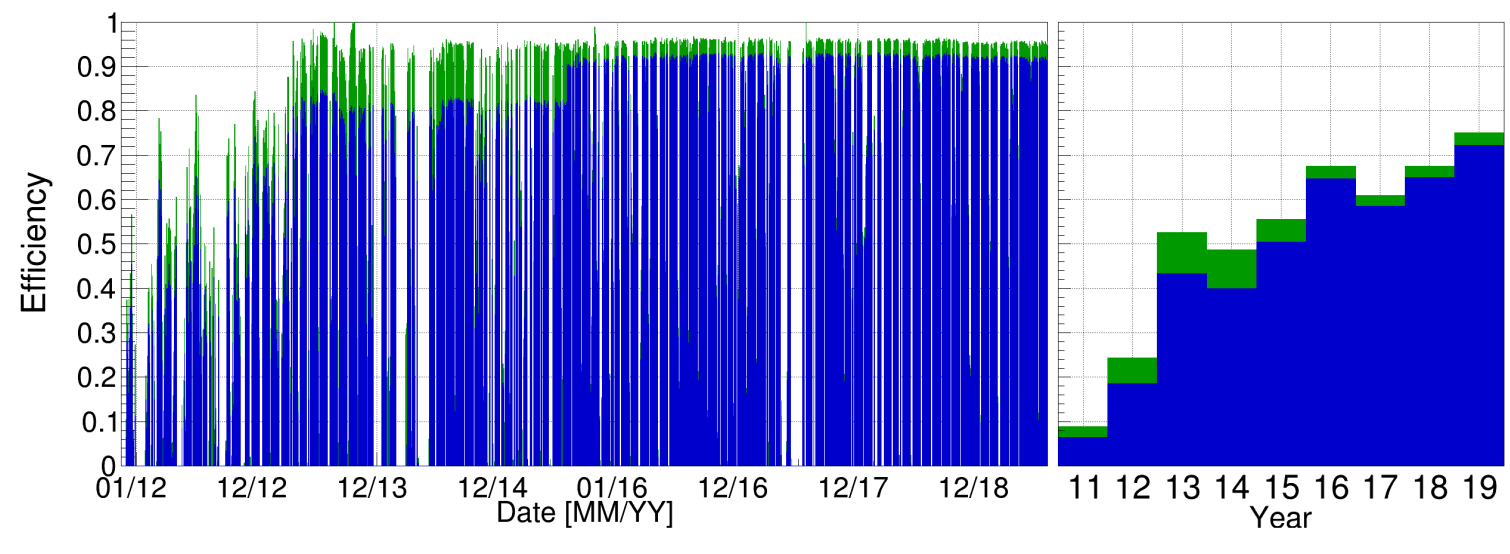

Figure 1: Total data taking efficiency per night (left) and average data taking efficiency per year (right), both normalized to the total time with the sun being at least $12^{\circ}$ below the horizon. Green indicates the sum of all recorded data and blue that of physics data. ${ }^{1}$

no dedicated calibration is needed. Therefore, in summer 2015, the amount of calibration data was reduced, further increasing the data taking efficiency for physics data to about $90 \%$ of the time with the sun being at least $12^{\circ}$ below the horizon (astronomical twilight). This is illustrated in Figure 1 (left). In the last 12 months $^{1}$, more than 2600 hours of physics data have been taken corresponding to $71 \%$ of astronomical twilight. This includes breaks due to bad weather, technical problems and a total of about 52 nights per year with no observations around full moon due to safety regulations. As, on average, La Palma provides $42 \%$ astronomical twilight, this correspond to an overall duty cycle of $35 \%$ per year assuming no shutdown time due to safety regulations.

Observing Strategy Furthermore, the observing strategy of FACT is focused on long-term monitoring: A small sample of sources is observed with a high cadence. An unbiased procedure is ensured by an automatic scheduling [14]. Table 1 lists the source sample observed by FACT with the overall observation times ${ }^{1}$. The core program focuses on the brightest TeV blazars Mrk 421, Mrk 501 and 1ES 1959+650. While priority is given to the monitoring of these sources, also targetof-opportunity observations (not listed in Table 1) are carried out by FACT, if the schedule allows.

Unprecedented Data Sample The synergy of automatic operation, photosensors with stable and excellent performance and an observing strategy dedicated to long-term monitoring provides a large data sample for these sources allowing for unprecedented studies. For Mrk 421, more than 3000 hours have been observed so far, for Mrk 501 more than 2800 hours and for 1ES 1959+650 more than 1900 hours (see also Table 1). Whenever the observing conditions allow, these sources are observed each day between 40 minutes and 6 hours depending on their visibility.

\section{Highlights from FACT Long-Term Observations}

\subsection{Multi-Wavelength and Multi-Messenger Correlations}

Multi-wavelength correlation study on 5.5 years of Mrk 421 As discussed in detail in [15], we studied the MWL variability of Mrk 421 from December 2012 till April 2018. Between GeV 
Table 1: Observation times of of sources monitored by FACT in the time range from November 2011 to June 2019. ${ }^{1}$ Only sources with more than 1000 hours of observation time are listed. For completeness, also the total amount of hours with physics data is given for the full time of operation and for the last 12 months. ${ }^{1}$

\begin{tabular}{lrrr}
\hline \hline Source & total observation time & nights & average observation time \\
\hline \hline Mrk 421 & 3182 hours & 1089 & 2.9 hours/night \\
\hline Mrk 501 & 2866 hours & 1219 & 2.4 hours/night \\
\hline Crab Nebula & 2286 hours & 926 & 2.5 hours/night \\
\hline 1 ES 1959+650 & 1918 hours & 947 & 2.0 hours/night \\
\hline 1 ES 2344+51.4 & 1751 hours & 866 & 2.0 hours/night \\
\hline 1H0323+342 & 1110 hours & 552 & 2.0 hours/night \\
\hline \hline all sources & $\mathbf{1 3 8 3 0}$ hours & $\mathbf{1 9 9 8}$ & - \\
\hline all (last 12 months) & $\mathbf{2 6 0 2}$ hours & $\mathbf{3 1 0}$ & - \\
\hline \hline
\end{tabular}

and radio emission, a time lag is found which can be modelled. This allows to predict the delayed radio emission from the measured $\mathrm{GeV}$ flux, favouring a leptonic scenario. While no correlation between $\mathrm{GeV}$ and $\mathrm{TeV}$ emission is found, there exists a strong correlation between $\mathrm{TeV}$ and $\mathrm{X}$-ray without significant time lag.

Exceptional activity of 1ES 1959+650 In summer 2016, 1ES 1959+650 showed an enhanced flux over few months with several bright outbursts [16, 17, 18, 19, 20]. Assuming a one-zone leptonic scenario, the low-energy bump is fitted. The high energy emission is calculated from this model. While low and intermediate flux states are well reproduced, the two brightest outbursts show a higher $\mathrm{TeV}$ flux than predicted from the model. This could hint a second emission region or mechanism [21]. Consequently, a search for neutrinos was performed using the gamma-ray light curves. However, no neutrino signal was found in temporal or spatial coincidence [22].

Target-of-Opportunity Programs and Flare Alerts The FACT Collaboration issues alerts to other instruments. According to the brightness of the sources, different trigger criteria have been set for different sources. Within the gamma-ray community, alerts are issued for a flux of more than three times the flux of the Crab Nebula at TeV energies (Crab Units (CU)) for Mrk 421 and Mrk 501. For all other blazars, the trigger limit is at $0.5 \mathrm{CU}$. The FACT Collaboration also sends these alerts to interested colleagues from the MWL community. In case of exceptional outbursts or prolonged flaring activity, Astronomer's Telegrams (ATels) are issued. Table 2 summarizes these alerts and ATels sent by the FACT Collaboration.

FACT alerts triggered a study on Mrk 501 including FACT, Fermi-LAT and H.E.S.S. data (see below). In summer 2016, FACT alerted the MWL partners about a high state of the extreme HBL 1ES 2344+51.4 allowing for an unprecedented study of the high energy bump reported in [23].

In addition to alerts by email or ATels, target-of-opportunity programs have been set up with INTEGRAL and XMM-Newton in combination with Swift-XRT to study the temporal evolution of the SED [24]. Furthermore, additional Swift-XRT observations have been triggered based on the FACT monitoring program. Also triggers sent to X-ray satellites are summarized in Table 2. So far, four triggers have been issued in the context of granted proposals with INTEGRAL and 
Table 2: Flare alerts, requests for Target-of-opportunity (ToO) observations to X-ray satellites, Astronomer's Telegrams (ATels) and GCN Circulars sent based on the FACT monitoring program. ${ }^{1}$

\begin{tabular}{lrrrr} 
Source & Alerts & ToOs & ATels & GCN \\
\hline Mrk 421 & 29 & 13 & 5 & - \\
\hline Mrk 501 & 6 & - & - & - \\
\hline 1ES 1959+650 & 59 & 7 & 5 & - \\
\hline 1ES 2344+51.4 & 4 & 1 & - & - \\
\hline Target-of-Opportunity & - & - & 1 & 4 \\
\hline total & $\mathbf{9 8}$ & $\mathbf{2 1}$ & $\mathbf{1 1}$ & $\mathbf{4}$ \\
\hline
\end{tabular}

XMM-Newton. Follow-up observations took place in December 2015 [24] with INTEGRAL and in June 2019 both with INTEGRAL and XMM-Newton. These observations were complemented by monitoring and follow-up observations by Swift-XRT to allow for distinguishing between time lags and orphan flares.

In case external alerts are followed up with FACT, feedback to the MWL community is provided with a circular via the Gamma-ray Coordinates Network (GCN) or an ATel. For completeness, also GCN circulars and ATels related to follow-up observations are listed in Table 2.

\subsection{Variability Characteristics}

Temporal Behaviour of Mrk 501 Based on a gamma-ray data sample including data from FACT, Fermi-LAT and H.E.S.S., differences in the shape of the flux distributions in different wavelengths and on different time scales were studied. While Fermi covers the long-term variability, H.E.S.S. measures the short-term variability with high sensitivity. This is complemented by FACT, which covers both the long time scales with nightly binning and the short time scales during a bright outburst in the night of June 23rd to June 24th, 2014. While the long-term flux distributions at GeV energies show a normal behaviour, the flux distributions at VHE favour a log-normal behaviour [4].

Fractional Variability Using public MWL data from radio to $\mathrm{TeV}$ energies, the fractional variability of Mrk 421 and Mrk 501 was studied [25]. To take into account the different characteristics of the data samples, a systematic study was carried out determining a systematic uncertainty based on the incompleteness of the data samples. From more than five years of data, a different behaviour in fractional variability versus energy for Mrk 421 in comparison to Mrk 501 was not confirmed.

Periodicity The presence of a binary black hole could modulate the flux of an AGN periodically [26]. Hints of a periodic signal have been found from blazars [27, 28]. The long-term light curves from FACT are ideally suited for the search of periodicity at TeV energies. In [29], the light curves are corrected for observational effects that could fake periodicities. Furthermore, the window function is taken into account and a false alarm rate is calculated. The resulting light curves are analyzed with the Lomb-Scargle periodogram, showing a hint for a 332-day period for Mrk 501.

\subsection{Quiescent State of AGN}

As discussed in [30], the quiescent state of a source (if it exists) or an upper limit for it can be determined studying the distribution of flux states. Using more than $11^{\prime} 700$ hours of physics 
data from FACT, an upper limit for the quiescent state for Mrk 421 of $22 \%$ of the flux of the Crab Nebula at $\mathrm{TeV}$ energies was determined [30]. The light curve of Mrk 501 shows a low state of the source since 2016. Using only data from the last three years of data taking, the upper limit for the quiescent state for Mrk 501 is improved to $2 \%$ of the flux of the Crab Nebula at TeV energies [30].

Alerts based on Definition of Quiescent State FACT participates in the astrophysical multimessenger observatory network (AMON) [31]. To issue sub-threshold alerts to AMON, the flux distribution is divided into a quiescent and active state [30]. Combining information of real-time alerts from different messengers, AMON issues multi-messenger alerts based on a correlation analysis.

\section{Summary}

Combining the advantages of SiPMs as photosensors with an observing strategy optimized for long-term monitoring, FACT has collected an unprecedented data sample for the brightest blazars at $\mathrm{TeV}$ energies. This monitoring complements the short deep exposures of high-sensitivity instruments and furthermore provides them with alerts in case of interesting events like bright flares. Based on these alerts and in combination with monitoring at other wavelengths, unprecedented studies are carried out.

\section{References}

[1] F. Aharonian, A. G. Akhperjanian, A. R. Bazer-Bachi et al., An Exceptional Very High Energy Gamma-Ray Flare of PKS 2155-304, 664 (2007) L71 [0 706 . 0797].

[2] V. A. Acciari, T. Arlen, T. Aune et al., Observation of Markarian 421 in TeV gamma rays over a 14-year time span, Astroparticle Physics 54 (2014) 1 [1310 . 8150].

[3] D. Dorner, J. Adam, L. M. Ahnen et al., FACT - Highlights from more than Five Years of Unbiased Monitoring at TeV Energies, ICRC 301 (2017) 609.

[4] C. Romoli, N. Chakraborty, D. Dorner et al., Flux Distribution of Gamma-Ray Emission in Blazars: The Example of Mrk 501, Galaxies 6 (2018) 135 [1812. 06204].

[5] K. Gazeas, Long-Term Optical Monitoring of Blazars, Galaxies 7 (2019) 58.

[6] M. Backes, M. Böttcher and H. Falcke, Millimeter-wave Monitoring of Active Galactic Nuclei with the Africa Millimetre Telescope, arXiv e-prints (2019) arXiv:1906.08607 [1906.08607].

[7] D. Thompson, Fermi: Monitoring the Gamma-Ray Universe, Galaxies 6 (2018) 117.

[8] E. Angelakis, E. Angelakis, L. Fuhrmann et al., AGN Astrophysics via Multi-Frequency Monitoring of $\gamma$-ray Blazars in the Fermi-GST Era, vol. 427 of ASPC, p. 289, Oct, 2010, 0910.0643.

[9] D. Dorner, R. J. Lauer, FACT Collaboration et al., First study of combined blazar light curves with FACT and HAWC, vol. 1792 of AIPC, p. 050020, Jan, 2017, 1610.0662 7, DOI.

[10] D. Dorner, FACT Collaboration, R. Lauer and HAWC Collaboration, Joint analysis of TeV blazar light curves with FACT and HAWC, ICRC 301 (2017) 625 [1708 . 05451].

[11] H. Anderhub, M. Backes, A. Biland et al., Design and operation of FACT - the first G-APD Cherenkov telescope, JINST 8 (2013) P06008 [1304 .1710]. 
[12] A. Biland, T. Bretz, J. Buß et al., Calibration and performance of the photon sensor response of FACT — the first G-APD Cherenkov telescope, JINST 9 (2014) P10012 [1403.5747].

[13] M. L. Knoetig, A. Biland, T. Bretz et al., FACT - Long-term stability and observations during strong Moon light, arXiv e-prints (2013) arXiv:1307.6116 [1307.6116].

[14] T. Bretz, A. Biland, J. Buß et al., FACT - Threshold prediction for higher duty cycle and improved scheduling, arXiv e-prints (2013) arXiv:1308.1516 [1308.1516].

[15] V. Sliusar and FACT Collaboration, FACT - Multi-wavelength analysis of more than 30 flares of Mrk 421, ICRC, These Proceedings 358 (2019) 796.

[16] S. Buson, J. D. Magill, D. Dorner et al., Fermi-LAT, FACT, MAGIC and VERITAS detection of increasing gamma-ray activity from the high-energy peaked BL Lac object 1ES 1959+650, ATel 9010 (2016) 1 .

[17] A. Biland, FACT measures increased gamma-ray flux from the high-energy peaked BL Lac object IES 1959+650 since five nights, ATel 9139 (2016) 1.

[18] A. Biland, D. Dorner, R. Mirzoyan et al., Further increase of gamma-ray emission from the HBL IES 1959+650, ATel 9148 (2016) 1.

[19] A. Biland and R. Mirzoyan, FACT and MAGIC measure an increased gamma-ray flux from the HBL IES 1959+650, ATel 9203 (2016) 1.

[20] A. Biland, FACT measures new maximum flux from the HBL 1ES 1959+650 at TeV energies, ATel 9239 (2016) 1.

[21] D. Dorner, FACT Collaboration et al., FACT - Time-Resolved Blazar SEDs, ICRC 301 (2017) 608.

[22] T. Kintscher, Icecube Collaboration, FACT Collaboration, MAGIC Collaboration et al., IceCube Search for Neutrinos from 1ES 1959+650: Completing the Picture, ICRC 301 (2017) 969.

[23] A. Arbet-Engels, M. Mangaranro, D. Dorner et al., Studying the Extreme Behaviour of IES 2344+51.4, ICRC, These Proceedings 358 (2019) 620.

[24] A. Kreikenbohm, D. Dorner, M. Kadler et al., Time-Resolved SEDs of Blazars Flares, in The X-ray Universe 2017, p. 119, Oct, 2017.

[25] B. Schleicher, A. Arbet-Engels, D. Baack et al., Fractional Variability-A Tool to Study Blazar Variability, Galaxies 7 (2019) 62.

[26] M. Tavani, A. Cavaliere, P. Munar-Adrover et al., The Blazar PG 1553+113 as a Binary System of Supermassive Black Holes, 854 (2018) 11 [1801.03335].

[27] M. Ackermann, M. Ajello, A. Albert et al., Multiwavelength Evidence for Quasi-periodic Modulation in the Gamma-Ray Blazar PG 1553+113, 813 (2015) L41 [1509.02063].

[28] G. Bhatta, Blazar Mrk 501 shows rhythmic oscillations in its $\gamma$-ray emission, 487 (2019) 3990 [1808.06067].

[29] FACT Collaboration, FACT - Probing the Periodicity of Mrk 421 and Mrk 501, International Cosmic Ray Conference, These Proceedings 358 (2019) 630.

[30] D. Dorner, A. Arbet-Engels, D. Baack et al., Flux States of Active Galactic Nuclei, Galaxies 7 (2019) 57.

[31] H. Ayala Solares, AMON Multimessenger Alerts: Past and Future, Galaxies 7 (2019) 19. 